\section{Pediatria Polska}

The editor and his board are to be congratulated for producing this new volume (with some help from a Western sponsor) of the journal of the Polish Paediatric Association after a long absence due to the difficult economic conditions in which eastern Europe finds itself today. The quality of the production and the variety and substance of the papers augurs well for the future of Polish paediatrics.

The volume of 100 pages contains a number of original papers (alas some submitted as long ago as two to three years), a handful of interesting case reports, a couple of reviews dealing with the use of corticosteroids in allergic disorders and the significance of virus infections in the pathogenesis of asthma respectively, and finally the journal ends with a report of an international meeting on the 'Rights of Children' and a brief historical summary of the development of oncological services for children in Poland.

The British paediatrician would not find a great deal to interest him/her-most of the articles are 'common or garden' stuff here. Possibly the best paper came from a dental faculty in Lublin, eastern Poland and dealt with the development of dental caries in insulin dependent diabetic children and controls, and which concluded that there were no significant differences in dentition in the two groups.

Surprisingly, many references are from English speaking countries, but regretfully most are outdated and from publications that are used in meeting sponsorships in the West. The attempts at brief summaries of the papers in the English language are, to put it charitably-awful and grossly inaccurate. It might be wise for the editors to consider writing in good English a detailed resume of one or two papers which they think may be of interest to the English speaking readers and confine the other submissions to English titles only.

Obviously this journal has taken first brave steps and has a long way to go in order to catch up with the publications in the West. Nonetheless, it was heartening to see so many submissions from various parts of Poland-a clear sign that the editors will not be short of clinical material in the future.

J A KUZEMKO

\section{LETTERS TO THE EDITOR}

\section{Hearing screening in children-state of the $\operatorname{art}(\mathbf{s})$}

SIR,-Haggard emphasised the extreme importance of early identification of hearing impairment in infants at risk of sensorineural hearing loss and advised evoked response audiometry as a sensitive and specific determinant. ${ }^{1}$ Reardon and Pembrey, in the same edition of the journal, documented that $30 \%$ of all genetically determined deafness occurs in syndromic form. ${ }^{2}$

Anatomical abnormalities of the inner ear and middle ear are well recognised in association with the head and neck syndromes and at present, imaging of the middle and inner ear is not routinely performed. Although screening tests would detect those infants with hearing impairment, for children with head and neck syndromes or external ear deformities it can only be complementary to imaging, which is the most important investigation, particularly for children in whom improvement is possible by surgical intervention. We believe that for all infants with syndromes associated with ear abnormalities-for example, Klippel-Feil, Treacher Collins, craniofacial microsomia, Wildervanck, Goldenhar, X linked deafnessor for infants with external ear deformities, that conventional tomography or high resolution fine section computed tomography in the neonatal period is essential, and that evoked response audiometry and tomography are complementary procedures. ${ }^{3}$

We have previously reported our series of 17 patients with Klippel-Feil syndrome who underwent hypocycloidal polytomography and axial computed tomography and in whom we found a high proportion of severe inner and middle ear abnormalities, many of which were incompatible with any degree of hearing (RR Phillips, PD Phelps, paper presented at the 50th annual meeting, Royal College of Radiologists, Liverpool 1989). In the inner ear there was a range of abnormalities, the most severe including dysplasia of the bony spiral of the cochlea (known as the Mondini defect), which occurred in six of our patients, or absence of any recognisable cochlear structure (the Michel deformity), which we demonstrated in five patients. There was a range of severity of middle ear abnormalities, which included abnormal ossicles in six patients, absent oval window in two, and small or slit attic in two.

Severe cochlear abnormalities are incompatible with auditory function and therefore bilateral abnormalities imply that education must use methods not involving sound. The Mondini defect allows some degree of cochlear function. For some patients with conductive deafness, surgical correction of the structural abnormalities may be successful in providing a degree of auditory function.

The radiological assessment of congenital deafness must assess inner ear structure, cochlear function, and the risk of cerebrospinal fluid fistula due to structural abnormalities. In the middle ear it must assess the feasibility of surgery for better sound conduction and the presence of any surgical hazards-for example, high jugular bulb. Any deformity of the bony labyrinth indicates a severe degree of sensorineural deafness and is therefore a relative contraindication to surgical attempts to improve the sound conduction mechanism.

Thus in patients with head and neck syndromes or external ear deformities, the early radiological assessment of the inner and middle ears (by conventional imaging and computed tomography in the neonatal period) is essential in order that the range of structural abnormalities may be demonstrated. At this stage imaging may be performed with little, if any, sedation and with a minimal radiation dose. Guidance may then be given regarding the possibility of corrective middle ear surgery and to allow the best methods of communication to be taught from the earliest opportunity.

RACHEL R PHILLIPS
PETER D PHELPS
Department of Imaging,
Royal Throat, Nose and Ear Hospital,
Gray's Inn Road,
London WCI

1 Haggard MP. Hearing screening in childrenstate of the art(s). Arch Dis Child 1990;65: state of

2 Reardon W, Pembrey $M$. The genetics of deaf ness. Arch Dis Child 1990;65:1196-7.

3 Lund VJ, Phelps PD and Beagley HA. Evoked response audiometry and tomographycomplementary procedures for the assessment of the deaf infant. Int $\mathcal{J}$ Pediatr Otorhino laryngol 1982;4:95-106. 